\title{
Une parenté de corps et un genre sexué : en dialogue avec Françoise Héritier
}

Des substances à la psyché, de la procréation à l'érotisme, de l'inceste du second type à l'inceste gémellaire, du ventre féminin au sperme masculin, de l'enfantement à l'engendrement, du Sahara à un centre de PMA

Corinne FORTIER

Chargée de Recherche première classe au CNRS (Laboratoire d'Anthropologie Sociale)

\begin{abstract}
Résumé : La pensée de Françoise Héritier relativement aux questions de substances, de différence des sexes, de procréation et d'inceste fera l'objet d'une analyse approfondie et sera mise en perspective avec mes propres recherches. Si Françoise Héritier a montré que le corps constitue le «socle» des représentations du masculin et du féminin, je démontre l'importance du corps comme support de représentations, non seulement du genre mais de la parenté.
\end{abstract}

Mots-clés : corps, genre, substances, inceste, procréation

1 La valence différentielle entre les sexes : et le genre?

J'essaierai de rendre compte de la pensée de Françoise Héritier (1933-2017) relativement aux questions de substances, de différence des sexes, de procréation et d'inceste, que je mettrai en perspective avec mes propres recherches entreprises dans la société maure de Mauritanie, société arabophone et musulmane, ainsi que dans un centre de procréation médicalement assistée du CECOS (Centre de Conservation des oufs 
et du Sperme humain) à Paris, terrain que j'ai entamé sous l'impulsion de Françoise Héritier $^{1}$.

Françoise Héritier n'utilise pas ou très rarement le terme de genre - elle parle de manière allusive des « catégories de genre » en 1991 dans un entretien avec l'anthropologue Nicole Échard (1997 : 91) —, préférant créer le concept de « valence différentielle des sexes » sans doute parce qu'à la différence du terme de genre ce concept inclut la différence des sexes et la notion de hiérarchie qui lui est coextensive, celle-ci s'étant efforcée de débusquer les ressorts de cette hiérarchie afin de mieux la «dissoudre» pour reprendre l'expression d'un sous-titre de ses livres (2002). Pour ma part, j'emploie le mot genre en prenant en compte également la différence des sexes, et en ne négligeant pas non plus la question de la domination masculine.

On connaît les résistances, y compris dans le milieu de la recherche, à adopter le terme de genre, qui n'est pas comme le disent ses détracteurs une «idéologie» - $c f$. les débats en France sur la prétendue «théorie du genre» - mais bien un concept heuristique. Le décentrement impliqué par ce concept relativement à la catégorie de sexe permet de complexifier l'analyse et d'éviter la confusion entre genre et sexe sans nier la réalité de la différence sexuée et son importance dans la construction même des catégories de genre.

Si les représentions genrées découlent de l'observation de certaines différences anatomiques entre les sexes comme cela est présupposé chez de nombreux auteurs qui ont traité de ce sujet (Freud, Roheim, Bettelheim, Devereux, Héritier, Godelier...), les types de différence anatomique privilégiés ainsi que les interprétations qui leur sont prêtées (avoir un pénis, un utérus, des menstrues, du sperme, des seins...) varient d'un auteur à un autre. Pour ma part, j'établirai que le genre est largement construit sur des représentations dérivées de l'observation du corps sexué même s'il ne s'y réduit pas (Fortier 2014a, 2014b, 2017a). Si Françoise Héritier (1996) a bien montré que le corps constitue le «socle» des représentations du masculin et du féminin, j'irai même plus loin relativement à l'importance du corps dans son aspect visible comme support de représentations, non seulement du genre mais de la parenté (Fortier 2009).

\section{Le corps féminin : et le corps masculin?}

Le corps, et la différence des sexes qui y est inscrite, est primordial pour Françoise Héritier dans la mesure où c'est de son observation que s'origine notre pensée structurée de façon dualiste en une série d'oppositions (droite/gauche, haut/bas, bien/mal, actif/passif, rationnel/sensible...) : «Système d'oppositions qui trouve son origine dans l'observation primale de la différence irréductible des sexes » (Héritier 2009 : 92). Françoise Héritier explique comment la différence anatomique des sexes sert de fondement à cette classification dualiste universelle — bien qu'elle préfère le terme d'invariant à celui d'universel, parlant à ce propos d' «armatures invariantes de la pensée » (2001 : 91) :

\footnotetext{
${ }^{1}$ Je souhaite ici rendre hommage à cette grande personnalité scientifique mais aussi morale avec qui j'ai eu des échanges intellectuels et amicaux, appartenant depuis 1998 à son équipe du Laboratoire d'Anthropologie Sociale.
} 
« J'ai abordé plus directement la question du rapport du masculin et du féminin dans un ouvrage rédigé à partir d'articles publiés dans des endroits épars, auxquels j’ai donné une cohésion et un sens général. Je situe dans le corps et dans son organisation visible, anatomique et physiologique, les bases du fonctionnement de la pensée à partir du classement entre identique à soi et différent de soi qui se serait opéré dans l'humanité émergente, compte tenu de l'obligation proprement humaine de donner du sens à ce qui entoure l'être dans le monde. L'être humain émergent ne pouvait le faire qu'avec les outils dont il dispose, à partir d'un regard posé sur ce qui lui était offert, son propre corps, le corps des autres, le corps des autres mammifères et également le cosmos. Un classement est donc opéré entre des corps semblables entre eux, et des corps différents des premiers. Une opposition majeure identique/différent, fondée sur la différence anatomique et physiologique du masculin et du féminin, organise et oriente à mon sens toute la pensée dualiste et hiérarchisée que l'on trouve dans l'ensemble des sociétés; cette opposition qui est nécessaire pour penser est hiérarchisée en raison de l'impossibilité de l'existence de l'équilibre. La question subsidiaire est alors celle de la raison d'être des valorisations positives et négatives qui sont accordées à chacun des pôles des catégories dualistes. Chaque pôle étant associé au masculin et au féminin selon les différentes cultures, le côté dévalorisé ou négatif est généralement féminin, le côté valorisé positif est masculin. On peut en faire la démonstration partout» (Héritier $1997: 71$ ).

Si la dichotomie classificatoire tient à la binarité sexuée, reste à expliquer la hiérarchie qui lui est coextensive, pour reprendre le terme de l'anthropologue Louis Dumont (1966), également employé par Françoise Héritier. Or, à la différence de Sigmund Freud (1856-1939), Françoise Héritier ne la situe pas dans le corps des hommes et dans le fait d'avoir ou non un phallus, mais dans le corps des femmes et dans sa capacité à produire des enfants — « pouvoir exclusif du sexe de la femme de donner naissance à un enfant » déjà relevé par Georges Devereux (1908-1985; 2011, or. 1983 : 189) —, pouvoir « exorbitant» que les hommes se réapproprient en imposant leur domination :

«En ce qui concerne le rapport du masculin et du féminin, Freud construit aussi la valorisation du masculin sur une définition anatomico-physiologique - l'absence ou la présence de pénis - et l'envie concomitante du phallus chez les femmes. Il m'a semblé, à travers l'analyse anthropologique que j'ai menée dans Masculin/Féminin, pouvoir montrer grâce à l'analyse de rituels et de mythes, mais aussi des représentations telles qu'elles s'expriment de la façon la plus quotidienne, que la domination passe plutôt par le contrôle et l'appropriation du pouvoir physiologique exorbitant qu'ont les femmes de donner naissance aux deux sexes, et corollairement par l'enfermement des femmes dans cette même fonction. L'omission du féminin dans la pensée freudienne s'exprime dans ces termes : le désir masculin d'appropriation du pouvoir physiologique de donner la vie me paraît ex- 
pliquer la domination plutôt que l'envie féminine du phallus » (Héritier $1997: 5)$.

Or cette « envie masculine» de s'approprier la capacité d'enfanter des femmes, avait été recouverte par Freud d'un «voile androcentrique» pour reprendre les termes de Bruno Bettelheim (1971 : 66), envie que celui-ci va mettre au jour (ibid. : 63), avant Françoise Héritier ou Maurice Godelier (1982), à partir de l'analyse de rituels d'initiation masculins comme la circoncision/subincision (Fortier 2020a : 51) ou la couvade (ibid. : 53).

De plus, Bruno Bettelheim (1971 : 167) avait souligné l'importance des menstrues féminines, regrettant que « dans son analyse subtile de quelques-uns des tabous les plus importants, Freud ait accordé si peu d'attention à ceux de la menstruation ». À son tour, Françoise Héritier (1985) prendra en considération cette «substance» féminine, repérant dans le fait que les femmes subissent chaque mois l'écoulement de leur sang, le signe visible de leur infériorisation et de leur assignation à l'ordre de la nature, à l'opposé des hommes qui se situent du côté valorisé de la culture, faisant, de leur propre chef, couler le sang à la chasse ou à la guerre - ou encore à la pêche (Fortier 2018d, 2018e, 2022b).

On peut s'étonner que Françoise Héritier ne mette pas en perspective cette substance féminine avec une autre substance masculine, le sperme, dans la mesure où comme la femme «voit» ses règles (Héritier 1985), l'homme «voit» son sperme. De plus, l'homme peut contrôler l'émission du sperme, à la différence du sang menstruel pour la femme. La polarité sperme-menstrues constitue donc un couple de substances opposées à partir duquel il est possible de lire l'opposition hiérarchique entre activité masculine (Fortier 2010) et passivité féminine, de même qu'on peut inférer cette opposition dans le fait d'avoir un phallus pénétrant et un vagin pénétrable. Mais Françoise Héritier n'évoque jamais le sperme en tant que substance différentielle, de même qu'elle ne cite jamais le phallus en tant qu'organe sexuel qui « fait la différence » selon Sigmund Freud, focalisant l'ancrage corporel de la valence différentielle des sexes non dans le corps masculin mais féminin.

Car la vraie différence des sexes pour Françoise Héritier, comme pour Bruno Bettelheim (1903-1990), ne réside pas dans le corps des hommes mais des femmes, et non seulement dans le fait d'avoir des menstrues, mais surtout dans la capacité d'enfanter, « pouvoir exorbitant» selon les termes de Françoise Héritier. Cette faculté anatomique qu'ont les femmes de faire des enfants dans leur corps est à la fois une force et une faiblesse d'après Françoise Héritier dans la mesure où elle les immobilise le temps de la grossesse et de l'allaitement, les rendant économiquement dépendantes des hommes :

«Le temps de la grossesse, au moins à son terme, et lors de l'allaitement qui peut durer deux ans ou plus dans certaines sociétés, la femme a une moins grande aptitude à la mobilité que l'homme et est ainsi cantonnée à la sphère du domestique et à la maternité ne peut vaquer à des activités économiques de cueillette ou d'élevage par exemple ce qui entraînerait une répartition des tâches» (1996: 231). 
Germaine Tillion (1907-2008), anthropologue qui reste peu citée dans les travaux sur le genre (Fortier 2019a), avait déjà avancé l'argument du lien de dépendance originel mère-enfant pour expliquer le fait que les femmes aient été, en tout temps et en tout lieu, cantonnées à leur rôle maternel :

«Les uns et les autres ont plus ou moins enfermé leurs femmes et les tiennent toujours à l'écart, mais les femmes s'en sont accommodées, car elles ont accaparé les enfants. Cette situation perdure depuis le néolithique... Bien que je ne pense pas que femmes de période antérieure aient eu une quelconque autorité - sinon, une fois vieilles, par l'intermédiaire de leur fils - car même aux époques très lointaines de préhistoire, les rapports de la mère et de l'enfant ont toujours été intenses, du fait que l'enfant n'est viable que lorsqu'il a dépassé sept ou huit ans. Donc, jusqu'à sept ou huit ans, il est en relation de dépendance totale avec les adultes qui l'ont engendré» (Tillion $1997: 33$ ).

\section{La fécondité comme fondement entre le masculin et le féminin : et l'érotisme?}

Françoise Héritier affirme dans Masculin, féminin (1996 : 230) que «ce n'est pas le sexe mais la fécondité qui fait la différence entre le masculin et le féminin ». Par voie de conséquence, elle remarque que, dans la plupart des sociétés, une femme ménopausée qui n'est plus féconde est assimilée à un homme, c'est du moins le cas chez les Samo du Burkina Faso où elle a mené son terrain. Or, dans la société maure de Mauritanie où j'ai conduit mes recherches, contrairement à ce qui a cours dans d'autres sociétés (Héritier 1996 : 25), la ménopause ne transforme pas une femme en homme et ne lui donne droit à aucune prérogative particulière.

De plus, dans ce même ouvrage, Françoise Héritier remarque (1996 : 103) que : «pour les hommes une femme ménopausée n'a généralement plus de valeur sur le plan de la séduction, car elle n'a plus le pouvoir de faire des enfants ». Mais dans la société maure de Mauritanie où la poésie masculine qui loue la beauté d'une femme tient une grande place (Fortier 2004b, 2021a, 2021b), ce n'est pas lorsqu'elle est ménopausée qu'une femme cesse d'être courtisée mais quand ses charmes se sont fanés. Ainsi, une femme maure perd sa féminité non pas quand elle ne peut plus procréer mais lorsque sa beauté s'est flétrie, c'est-à-dire quand le temps lui a ravi son pouvoir de séduction.

Si la fécondité féminine fonde la distinction entre le masculin et le féminin dans la mesure où le pouvoir d'enfanter est propre aux femmes comme l'a montré Françoise Héritier, on peut ajouter que la présence de charmes spécifiques susceptibles de séduire les hommes détermine également la féminité (Fortier 2022a). L'érotisme, caractéristique par excellence du féminin, se retrouve au-delà de la société maure dans de nombreuses sociétés, qu'il soit un attribut féminin largement partagé ou l'apanage d'une certaine catégorie de femmes (prostituées, courtisanes...).

Concernant l'érotisme, Françoise Héritier (1996 : 103) constate que « la ménopause met les femmes hors du désir et hors du regard ». Si j'ai pu nuancer cette affirmation, il n'en reste pas moins que, comme Françoise Héritier le suggère ici, c'est bien le 
regard de l'homme et corrélativement son désir qui fait la féminité d'une femme et qui la défait, ce que Laura Mulvey appelle le «male gaze». Ainsi, les femmes prises dans ce reflet d'elle-même performent (Butler 2005, or. 1990) ce que le regard masculin attend d'elles, accomplissant une certaine «mascarade», selon le terme introduit pour la première fois par la psychanalyste Joan Rivière (1994 : 176, or. 1929) et repris par Jacques Lacan (1973, or. 1964), qui renvoie à une mise en scène de la féminité. Selon mon hypothèse (2012a et 2021e), cette mise en scène de la féminité vise à susciter le désir des hommes sans réactiver leur angoisse de castration.

Par ailleurs, Françoise Héritier observe qu'une femme stérile connaît le même destin qu'une femme ménopausée, à savoir que, ne pouvant procréer et ayant par conséquent perdu ce qui caractérise sa féminité, elle accède à un statut de « quasi-homme » (1996 : 251). Or, là encore cette affirmation n'est pas universelle, car, en Mauritanie, la stérilité comme la ménopause ne transforme pas une femme en homme. De surcroît, dans la société maure, lorsqu'une femme est considérée comme stérile ${ }^{2}$, son mari ne la répudie pas ainsi que c'est le cas dans d'autres sociétés ${ }^{3}$.

Françoise Héritier ne prend sans doute pas assez en considération la question des affects qui existent non seulement dans les sociétés dites individualistes, pour reprendre l'expression de Louis Dumont (1966), mais également dans les autres sociétés. Comme j'ai pu le montrer (Fortier 2018a et 2021c), l'amour n'est pas, malgré les affirmations de Denis de Rougemont (1972), une création «occidentale» née au XII ${ }^{\mathrm{e}}$ siècle puisqu'il était déjà présent en Orient ainsi qu'en témoigne l'existence de poèmes d'amour préislamiques en Arabie ancienne. Tenter d'historiciser et de géographier l'apparition de l'amour est par ailleurs en soi une gageure étant donné qu'il s'agit d'un sentiment universel qui prend des formes culturelles variées dans le temps et dans l'espace. Cependant, un des «invariants» de l'amour réside dans le fait que son expression est avant tout une prérogative masculine (Fortier 2004a), les hommes étant des sujets désirants et les femmes objets de désir, caractéristique qui demeure une constante de la «valence différentielle des sexes» et qui est d'autant plus difficile à faire évoluer qu'elle relève de l'intime.

Même si Françoise Héritier fonde davantage la domination masculine dans le corps féminin, elle a néanmoins réintroduit le corps masculin dans sa dimension phallique pour expliquer les violences sexuelles faites aux femmes (harcèlement, viol, prostitution) qui reposent sur la représentation selon laquelle les pulsions sexuelles masculines ne doivent pas être entravées, élément qu'elle considère comme le plus fort et le plus constant de «la valence différentielle des sexes» (2002:310).

\footnotetext{
${ }^{2}$ Même si en l'absence de tests médicaux, on ne peut vérifier lequel des deux membres du couple est infertile dans la mesure où l'infertilité est d'emblée envisagée comme provenant de la femme et non de l'homme comme l'a bien noté Françoise Héritier.

${ }^{3}$ Avoir une descendance étant très important dans la société maure, c'est la seule raison qui peut conduire une femme ayant exigé de son mari la monogamie dans son contrat de mariage (Fortier 2011a) à accepter exceptionnellement la polygamie.
} 


\section{Le pouvoir d'enfantement des femmes : et la puissance d'engendrement des hommes?}

Alors que Françoise Héritier associe davantage la fécondité à la femme qu'à l'homme, mes recherches témoignent que non seulement l'enfantement est très important dans la construction de la féminité, mais également l'engendrement du point de vue masculin. En effet, de nombreuses sociétés valorisent le fait d'avoir «beaucoup d'enfants » selon la formule d'usage connue dans notre société, pour des motifs sociaux, économiques et affectifs mais aussi, ce qui est moins mis en avant, pour des raisons liées à l'identité de genre, dans la mesure où avoir de nombreux enfants participe pour un homme de sa virilité.

Ce fait est particulièrement visible dans les sociétés " non occidentales », par exemple dans la société maure de Mauritanie où il est flatteur pour un homme de voir ainsi souligner l'importance de sa progéniture masculine : « Vingt hommes sont issus de son dos ». Si ce type d'expression atteste de sa bonne fortune, elle témoigne également de sa virilité ; dans cette société comme dans d'autres, la référence au dos, partie pérenne et dure du corps, représente d'une part la lignée agnatique qui forme l'ossature de la structure sociale et constitue d'autre part le lieu de production de la substance fécondante qu'est le sperme, ce qui montre l'indissociabilité au niveau des représentations du social et du biologique (Fortier 2001).

Avoir une progéniture importante représente pour un homme un élément de prestige en ce qu'elle lui permet de perpétuer sa lignée, et elle constitue aussi une source de fierté masculine dans la mesure où l'homme manifeste de cette manière sa puissance sexuelle. Ainsi, le phénomène d'hyperfertilité peut-il être interprété de façon distincte lorsqu'il concerne des hommes ou des femmes : à l'homme qui a de nombreux enfants sera généralement reconnu un rôle culturel et civilisationnel en tant que reproducteur de la société, alors que pour la femme, même si cela est aussi socialement valorisé, un tel fait sera associé à l'animalité et à l'excès (ubris) relevant de l'ordre de la nature.

Et, lorsque certaines sociétés - les sociétés occidentales par rapport à d'autres sociétés — ou certaines classes sociales — les «bourgeois»versus les 《prolétaires 》dont l'étymologie latine (proles) renvoie au fait de procréer — affirment, par distinction avec d'autres populations qu'ils considèrent comme moins développées et moins civilisées, que celles-ci se reproduisent à la manière d'animaux, ces populations sont rejetées du côté de la nature, de la féminité et de la non-maîtrise sexuelle, a contrario des populations dominantes qui se situent du côté valorisé de la culture, de la masculinité et de la maîtrise sexuelle.

Cette différence de perception entre l'hyperfertilité masculine et féminine tient sans doute au fait que la fertilité est indissociable de la sexualité, or la sexualité de la femme a un statut différent de celle de l'homme dans la mesure où elle peut aboutir à une naissance ; la sexualité féminine en tant qu'elle concerne «l'ordre reproductif » du groupe se doit donc d'être contrôlée par celui-ci. Entre autres formes de contrôle, la chasteté, sinon la virginité, qui est imposée aux femmes dans de nombreuses sociétés, contraste avec l'attitude attendue des hommes pour qui le fait d'avoir de nombreux enfants est comparable au fait d'avoir de multiples partenaires sexuels, comme si la 
fertilité masculine était indissociable de la virilité. Il est par ailleurs significatif que dans certaines sociétés, notamment arabes, où l'impératif de chasteté au mariage est très important, le drap taché de sang de la mariée est non seulement rattaché à la femme et à sa virginité, mais à l'homme et à sa puissance phallique (Fortier 2012a). Ce qui vient confirmer, que, dans de nombreuses sociétés, c'est moins la préservation de la virginité de la femme qui est essentielle, ainsi qu'on l'affirme haut et fort (Fortier 2021d), que tout ce qui peut éviter à l'homme la manifestation de son angoisse de castration et le maintien de sa puissance virile, motivation qui, en revanche, est indicible (Fortier 2021e).

\section{Du phallus en érection : et le sperme fécond?}

De même que, de façon quasi universelle, le symbole viril est un phallus, soit un pénis qui ne peut être représenté dans les figurations picturales ou imaginaires qu'en érection, le sperme, autre insigne du masculin, ne peut être pensé que comme fécond, ainsi que le montre l'image bien connue, dans de nombreuses sociétés, de l'eau, métaphore du sperme, qui, en tombant, vient féconder une terre généralement considérée comme féminine. Du fait de cette association entre virilité, fécondité et puissance phallique - Françoise Héritier (1996 : 77) remarque que les Samo du Burkina disent suggestivement de l'homme infertile que «son pénis est mort» -, toute faille dans l'érection ou l'infertilité est assimilée à de l'impuissance sexuelle. De même, dans le cadre des procréations médicalement assistées, l'annonce de son infertilité pour un homme s'apparente à un traumatisme (Fortier 2005), car comme l'a bien vu Françoise Héritier (1996 : 77), la stérilité de l'homme est inextricablement associée à son impuissance et représente donc une atteinte à sa virilité. Bien qu'infertilité et impuissance constituent dans les faits deux réalités dissociables, elles demeurent indissociables du point de vue des représentations communes.

Lors de mes recherches dans un CECOS qui pratique le don de gamètes, j'ai pu observer qu'afin de masquer leur impuissance à procréer, faussement associée à l'impuissance sexuelle, certains hommes préfèrent que leur couple ait recours au don de sperme plutôt qu'à l'adoption, craignant que l'enfant adopté, de par sa dissemblance possible (Fortier 2011c et 2001d), ne révèle au grand jour leur stérilité. La grossesse d'une femme survenue à la suite d'une «Insémination Avec Donneur (IAD)» étant spontanément attribuée à son époux, cette pratique permet que les apparences soient préservées quant à la virilité et à la paternité biologique du mari.

Parmi les couples que j'ai rencontrés dans le cadre de cette étude, la femme apparaît comme moteur relativement au projet d'enfant, mais le choix de sa réalisation par le moyen des procréations médicalement assistées plutôt que par celui de l'adoption revient le plus souvent à l'homme. En dépit de ce que leur épouse puisse devenir enceinte à partir du sperme d'un autre qu'eux, l'IAD permet à ces hommes de croire et de faire croire que la grossesse est de leur fait, ainsi que le laisse supposer la formulation ambiguë qu'ils utilisent à ce sujet : " Je veux offrir une grossesse à ma femme ». Plus généralement, la virilité de l'homme réside aussi bien dans le fait de rendre une femme enceinte que de lui procurer de la jouissance, le corps féminin constituant le lieu d'expression de sa puissance phallique. La grossesse de la femme 
est de fait attribuée à l'homme, comme le montrent certaines expressions que l'on retrouve dans différentes sociétés dont la nôtre telles qu' « il l'a mise enceinte » ou « il l'a engrossée », ou encore, quand la grossesse aboutit à une naissance, « il lui a fait un enfant ». En l'occurrence, c'est le sperme qui est considéré comme la cause première de la transformation du corps féminin en un corps fécond ${ }^{4}$.

\section{Le ventre des femmes : et le sperme des hommes?}

Françoise Héritier remarque dans Masculin, féminin (1996 : 121) : « Toutes les formes de stérilité, ont toujours été considérées dans les représentations collectives comme étant d'origine purement féminine, comme procédant du mauvais vouloir de la féminité ». Mes travaux sur les procréations médicalement assistées en France m’ont montré que ce constat est toujours d'actualité dans le contexte ultra-médicalisé du don de gamètes où les représentations « archaïques » d'un sperme nécessairement fécondant et d'un ventre possiblement stérile demeurent primordiales malgré les connaissances scientifiques et médicales.

Dans le cas d'un problème de fertilité qui vient de l'homme, il a l'avantage d'avoir été diagnostiqué par les médecins, tandis que de son côté, la femme objectivement fertile qui n'arrive pas à être enceinte après plusieurs inséminations (IAD) se retrouve confrontée à un non-sens. Les femmes se sentent coupables de ces échecs inexpliqués d'autant plus que le sperme inséminé ne peut en être à l'origine puisqu'il est censé avoir été sélectionné selon des critères d'exigence supérieurs à ceux qui caractérisent habituellement un sperme fécond.

Le terme médical employé pour qualifier ces femmes qui ont subi sans succès un traitement hormonal, celui de «mauvaises répondeuses », semble ainsi faire écho à la vieille idée quelque peu téléologique selon laquelle le corps féminin pourrait insidieusement s'opposer à ce pourquoi il est fait, l'enfantement, idée bien mise en exergue par Françoise Héritier (1996 : 81) ; opposition apparaissant dans ce contexte médical comme d'autant plus puissante qu'elle est capable de tenir en échec les traitements hormonaux qui ont pour fonction de stimuler la fécondité féminine. Il existe en effet une ambivalence attribuée au pouvoir procréateur de la femme, le corps féminin apparaît pourvu d'attributs contraires : le giron, lieu favorable au développement du foetus, est aussi perçu comme un ventre inhospitalier où, selon l'expression récurrente employée par les femmes ayant recours à l'aide médicale à la procréation, « cela ne prend pas ».

\section{Invisibilité de l'ovocyte versus visibilité du sperme : une conception spermatique?}

D'après mes recherches, il apparaît que même dans le cadre scientifique et médicalisé des PMA, alors qu'on sait que les gamètes des deux sexes sont nécessaires pour fabriquer un enfant, la part de la femme dans la reproduction de la vie se réduit à sa fonction de réceptacle, fonction féminine par excellence, qui vient occulter sa participation générative au profit de celle de l'homme. L'opposition existant entre

\footnotetext{
${ }^{4}$ Cette idée peut être retrouvée à des étapes antérieures à celle de la conception, par exemple, chez les Baruya de Nouvelle Guinée où le sperme ingéré par la jeune épouse permet de développer sa fécondité (Godelier 1982 : 363).
} 
l'invisibilité de l'ovule qui tient à son caractère interne et la plus grande visibilité du sperme expliquerait que l'existence de l'ovocyte ne puisse être pensée, y compris par les femmes elles-mêmes. Que représente en effet un ovocyte pour une femme? Élément de fertilité, il ne peut être vu, à la différence du sang menstruel ${ }^{5}$, sang qui signale $a$ contrario à une femme qu'elle n'est pas enceinte.

Cependant, cette occultation du rôle d'une substance féminine dans la conception est d'autant plus paradoxale que les procréations médicalement assistées déclenchent au moyen d'un traitement hormonal la production de plusieurs ovocytes chez la femme, et non plus un seul comme dans un cycle naturel, ce traitement pratiqué avant l'insémination visant à multiplier les chances de fécondation et de grossesse. L'importance accordée à l'ovocyte est aussi accentuée par les progrès des techniques de visualisation, et en particulier de l'échographie, qui, en rendant visibles les follicules ovariens, donnent une plus grande valeur de réalité à ce qui restait auparavant invisible et donc quasi inexistant. Il apparaît néanmoins que cette représentation oviste induite des techniques médicales de procréation coexiste avec une représentation spermatique plus courante et plus prégnante de la conception.

On sait en effet que les connaissances scientifiques peuvent cohabiter sans trop de contradiction dans l'esprit humain avec des représentations collectives ou des croyances culturelles. Ainsi, dans un entretien avec Philippe Girard, Françoise Héritier (2009 : 195) remarquait l'archaïsme de certains discours prétendument médicaux sur la fécondation :

\begin{abstract}
« [...] dans un article médical écrit par de grands spécialistes, se trouvait une analyse de la fécondation dont il était très facile de montrer que les termes utilisés pour décrire le rôle des gamètes renvoyaient au modèle archaïque du rapport entre les sexes : l'inertie fondamentale de la cellule féminine fait qu'elle est condamnée à mourir sans la "puissance activante" et la "vertu" séminale du gamète mâle. Le fait même d'utiliser ce langage renvoie à un système de représentation extrêmement archaïque ».
\end{abstract}

La force de cette théorie spermatique de la conception tient, selon mon analyse, à son évidence du point de vue des sens en tant qu'elle se fonde sur la représentation la plus immédiate du rapport sexuel, dont chaque individu de toute société sait qu'il est à la base de la grossesse d'une femme et de la naissance d'un enfant (Héritier 2004 : 101), représentation où l'homme joue un rôle actif dans la conception de par l'émission de sperme.

Ainsi, dans notre société, où la fonction de l'ovule dans la procréation est connue et où elle peut même revêtir une importance primordiale dans les procréations médicalement assistées, le rôle du sperme à l'étape de la conception reste prégnant. Quel que soit le système de filiation, la plupart des sociétés considèrent que le sperme masculin joue un rôle dans la reproduction, alors qu'elles peuvent n'accorder aucune fonction

\footnotetext{
${ }^{5}$ L'expression même de «voir », selon Françoise Héritier (1985), était utilisée par les paysannes en France pour signifier qu'elles avaient leurs règles.
} 
substantielle à la mère ${ }^{6}$. En outre, de nombreuses sociétés qui reconnaissent l'existence de deux substances sexuées, l'une masculine et l'autre féminine, peuvent néanmoins attribuer à l'étape de la conception un rôle majeur à la substance masculine et un rôle mineur à la substance féminine. La survalorisation de la participation masculine lors de la conception tient, selon mon hypothèse, au fait que l'homme ne joue aucun rôle physiologique visible lors des autres étapes du processus de procréation. Le sperme, qui apparaît comme l'unique apport physiologique masculin dans la fabrication d'un enfant, est alors survalorisé relativement à sa fonction générative.

Françoise Héritier (2009 : 81) remarquera ce primat du sperme dans la procréation : «Je ne pense pas quoi qu'on en ait dit $^{7}$, qu'il y ait jamais eu une société n'ayant pas fait le lien entre rapport sexuel, sperme et procréation ». L'homme est incontestablement nécessaire pour «engrosser » la femme; même dans le cas où des sociétés considèrent que la femme peut porter en son sein un enfant conçu par un esprit, celui-ci paraît être toujours masculin (souffle spermatique, ancêtre...). Ainsi, certaines théories de la conception semblent faire la part belle à l'homme, afin de justifier la contradiction selon laquelle, en dépit de l'importance de la femme dans le processus de génération, le produit en revient à un parent de sexe masculin, qu'il s'agisse du père, dans une société patrilinéaire, ou de l'oncle maternel, dans une société matrilinéaire. Ces théories masculines de la conception viennent, selon mes conclusions, contrebalancer le constat incontournable selon lequel le corps de la femme est nécessaire pour générer un être humain. Le fait que la substance fécondante soit dans la plupart des sociétés essentiellement masculine participe de ce que Françoise Héritier, comme Maurice Godelier (1982), ont identifié comme l'appropriation imaginaire par les hommes du pouvoir essentiellement féminin de produire une descendance.

De surcroît, l'étude d'autres sociétés que la nôtre révèle que la survalorisation du rôle de l'homme dans la fabrication d'un enfant concerne non seulement l'étape de la conception, mais aussi les autres étapes procréatives : par exemple, la gestation, lorsque le fœetus est censé se développer sous l'action bénéfique du sperme apporté par les rapports sexuels que l'homme a avec son épouse pendant qu'elle est enceinte (Fortier 2001), ou encore, le fait de porter et d'accoucher de l'enfant, ainsi que le montrent les rites de couvade où l'époux peut connaître un régime alimentaire et des douleurs semblables à la femme au moment où elle est enceinte et accouche, ou enfin, l'allaitement, quand le lait donné au nourrisson censé provenir du sperme de l'époux achève de former le corps de l'enfant et crée des interdits matrimoniaux comme c'est le cas en islam (Fortier 2001, 2007, 2011b, 2018b) ${ }^{8}$ qui reconnaît la parenté de lait (Héritier 1994) ${ }^{9}$.

\footnotetext{
${ }^{6}$ Comme le souligne Françoise Héritier (1996 : 136), cette « exclusivité paternelle », selon laquelle la mère se contente de prêter son corps au développement du foetus, ne se retrouve pas seulement dans des sociétés patrilinéaires, mais également dans des sociétés matrilinéaires ou cognatiques.

${ }^{7}$ Françoise Héritier fait référence à l'anthropologue Bronislaw Malinowski selon lequel les Trobriands, et plus généralement les «sociétés primitives", auraient ignoré le rapport causal entre le fait d'avoir des relations sexuelles et le fait de procréer (Héritier $2004: 101$ ).

${ }^{8} \mathrm{~J}$ 'ai été la première anthropologue française à étudier la parenté de lait en islam du point de vue des textes musulmans (Coran, Sunna, textes juridiques et médicaux) ainsi qu'à partir de terrains de recherche, après avoir rencontré cette forme de parenté en Algérie (1998) puis dans la société maure de Mauritanie (2001).

${ }^{9}$ Françoise Héritier (1994 : 309-325) s'est intéressée à la parenté de lait dans le monde musulman compte tenu de la disproportion entre le nombre élevé de prohibitions matrimoniales qui en relèvent comparé au
} 
Or, si la représentation essentiellement spermatique de la conception participe de l'appropriation masculine du pouvoir de fabriquer un enfant, elle s'en distingue néanmoins sur un point important; le processus d'accaparement par les hommes des capacités féminines liées à la gestation, à l'accouchement et à l'allaitement est totalement imaginaire et va même à l'encontre de l'évidence des sens, puisqu'à toutes ces étapes, le rôle de la femme est incontestable : c'est elle qui est enceinte, donne naissance à l'enfant et le nourrit de son lait, en revanche, la lecture masculine de l'étape de la conception n'est pas complètement décalée de la réalité dans la mesure où elle peut trouver une confirmation dans l'observation du rapport sexuel, où l'émission de sperme est perceptible. Le primat du masculin dans la conception, qui existe aussi dans notre société, s'expliquerait notamment par le fait que l'ovocyte est imperceptible en comparaison de la visibilité du sperme masculin dans les rapports sexuels.

Ainsi, la survalorisation masculine lors de la conception, constatée dans beaucoup de sociétés, y compris dans la nôtre, tiendrait donc au fait que l'homme joue un rôle physiologique visible à ce moment de la production d'un enfant, et non aux autres étapes que constituent la grossesse, l'accouchement et l'allaitement, hormis de façon imaginaire. Ce fait universel expliquerait que de nombreuses sociétés accordent un rôle majeur au sperme de l'homme et ce indépendamment de leur mode de filiation, patrilinéaire ou non comme l'a souligné Françoise Héritier (1996 : 104) :

« Dans bien d'autres sociétés, matri- comme patrilinéaire ou cognatiques d'ailleurs, on trouve cette exclusivité paternelle, la mère se contentant, selon des figures métaphoriques explicites, de prêter son corps au développement du foetus, telle une besace que l'on remplit ou une poterie où se concocte une cuisine de choix ».

Il semble donc que le rapprochement anthropologique qui a pu être fait entre les conceptions relatives à la fabrication d'un enfant et les systèmes de parenté ne rend pas compte d'une certaine constance des représentations du rôle fécondant du sperme masculin et du rôle récepteur du corps féminin, constance qui, à travers le rapport sexuel, tient, selon moi, à la plus grande visibilité de la substance masculine, interprétée comme cause essentielle de la conception.

\section{Un inceste de substances : et l'inceste psychique?}

Françoise Héritier a relié la question de l'inceste à la circulation des substances entre personnes apparentées. C'est dans cette perspective qu'elle crée le concept d'inceste du second type soit «la mise en rapport de deux consanguins de même sexe qui partagent le même partenaire sexuel » (1994 : 12). Françoise Héritier, s'en tenant au cadre épistémologique propre à la discipline anthropologique, a ignoré la dimension psychique de l'inceste, comme elle l'explique au psychanalyste Jacques André :

« L'horreur, cette horreur qui est socialement construite, qui a une histoire fondée sur des règles transmises, ne veut pas dire pour autant qu'il ne peut 
y avoir de délectation à vivre l'inceste, dans le fantasme ou dans la réalité. S'il n'y avait pas de délectation, de désir, l'inceste n'existe pas, or il existe. Il y a un rapport évident entre l'interprétation anthropologique et l'observation psychanalytique, même si je ne me suis jamais consacrée à mettre en évidence les liens existants. La psychanalyse parle en termes de désir, de relation fusionnelle. L'anthropologie sociale en reconnaît l'importance pour les individus mais s'intéresse aux représentations partagées touchant à l'acte, aux substances, à la naissance du lien naturel qui unit ceux qui partagent une même consanguinité, à la nature du rapport sexuel, à celle des substances impliquées dans cet acte. C'est la façon anthropologique de voir les choses. Je pense qu'une théorie unifiante devrait prendre en compte les deux approches [...]»(Héritier 2001 : 110).

Ma propre démarche théorique qui combine anthropologie et psychanalyse correspond d'une certaine manière à la «théorie unifiante» appelée de ses vœux par Françoise Héritier.

Concernant les substances procréatives dont parle Françoise Héritier, j'ai montré que ces substances ne doivent pas nécessairement entrer en contact par le moyen d'un rapport sexuel pour qu'il y ait inceste (Fortier 2005), mais que celui-ci peut résulter de leur rencontre in vivo ou in vitro comme c'est le cas dans le cadre des techniques médicales de procréation. Celles-ci, en effet, en excluant le rapport sexuel et en apparaissant comme un échange de substances ayant pour unique but la procréation, n'éliminent pas pour autant les multiples dimensions de la sexualité qui interviennent au contraire ici dans toute leur complexité.

D'une part, ces formes de procréation ne peuvent oblitérer les représentations sexuées attachées à certaines substances comme le sperme, ou à certains actes comme l'insémination. D'autre part, ces pratiques procréatives, parce qu'elles ont recours à un tiers, peuvent susciter des fantasmes d'inceste dans la mesure où ces inclinations, quoiqu'interdites socialement ou moralement, à des degrés différents, peuvent aussi être inconsciemment désirées. C'est en effet un des apports fondamentaux de la psychanalyse que d'avoir montré que la sexualité, et l'aspect incestueux qu'elle peut revêtir, ne réside pas seulement dans l'acte sexuel mais au niveau du désir. Cette conception non plus agie mais psychique de l'inceste est très importante pour comprendre les enjeux sociaux et intimes que soulèvent les procréations avec don (Fortier 2020b).

Une fois qu'on a admis que la sexualité n'est pas restreinte à l'acte sexuel procréatif, mais s'étend à la circulation des substances telles que le sperme dont la valeur est à la fois sexuelle et procréative, et qu'elle est également nichée, de façon moins « physique » mais non moins « réelle» dans nos pensées les plus inconscientes, il est clair que la sexualité n'a pas disparu des techniques médicales de procréation, et ce malgré l'idée communément admise que celles-ci ont opéré une disjonction entre procréation et sexualité.

Mes recherches montrent en effet que, loin d'avoir évacué la sexualité, celle-ci est réactivée de façon complexe par les procréations médicalement assistées avec tiers donneur dans la mesure où elles ne concernent pas seulement un couple mais impliquent trois 
personnes, souvent apparentées, deux de même sexe et une personne de sexe opposé, fournissant de multiples situations d'inceste et réactualisant le jeu des identifications et des désirs incestueux du triangle odipien (Fortier 2017b, 2019d).

\section{Inceste du second type : et l'idéal de ressemblance?}

Certains des dons directs de gamètes entre parents relèvent de l'inceste, et notamment de l'inceste du deuxième type, ainsi nommé par Françoise Héritier (1994) pour le distinguer de l'inceste du premier type dont le caractère premier tient à sa plus grande évidence. Le paradigme de l'inceste du deuxième type met en contact substantiel deux sœurs, ou une fille et une mère, par l'intermédiaire du sperme d'un même homme; cela correspond à des situations connues dans le don d'ovocyte, lorsque le don entre sœurs fut pratiqué en France, ou encore celui de fille à mère, comme cela existe aux États-Unis ${ }^{10}$.

Le don entre parents a pu être envisagé dans la mesure où les avantages apportés, notamment la transmission d'un capital génétique commun, apparaissaient comme plus immédiats et plus tangibles que la conscience de l'inceste qu'un tel don implique. C'est ce que montre le cas de Jeanine Salomone (2002 : 43) qui défraya la chronique en France en 2001 en donnant naissance, à plus de soixante ans, à un enfant conçu par don d'ovocyte grâce au médecin italien Antinori, confirmant l'importance que revêt les ressemblances pour que l'enfant s'inscrive dans une lignée :

«Je souhaite que mon enfant soit un Salomone. Qu'il me ressemble par certains traits physiques et moraux qui seront nos tendres repères à tous les deux. Que je me retrouve en lui comme il pourra se retrouver en moi. Qu'il fasse partie de nous, qu'il soit une partie de moi. Mon frère ayant les mêmes gênes que moi, je songe à lui pour être le donneur de sperme. Ne sommes-nous pas issus de la même mère et du même père? ».

Cette recherche obsessionnelle de ressemblance (Fortier 2012b) amène Jeanine Salomone à avoir recours au don de sperme de son frère sans qu'elle semble en envisager le caractère incestueux. Pourtant, comme je l'ai déjà montré, ce n'est pas parce que les procréations médicalement assistées sont indépendantes de l'acte sexuel, qu'elles ont perdu toute dimension sexuelle et incestueuse (Fortier 2005 : 60-61). En l'occurrence, la volonté de partager avec son enfant le même patrimoine génétique ainsi que des caractéristiques semblables est dans le cas de Jeanine Salomone si forte que le caractère incestueux inhérent au choix d'avoir un enfant de son frère est alors dénié.

En outre, cette recherche de ressemblance à tout prix, y compris au prix d'un inceste, se situe à l'opposé de la conception trobriandaise étudiée par Bronislaw Malinowski en Mélanaisie où l'on évite de souligner la ressemblance éventuelle d'un enfant avec son oncle maternel puisqu'elle serait interprétée comme un manquement au tabou de l'inceste entre frère et sœur. L'anthropologue précise dans La vie sexuelle des sauvages $(1930: 136)$ :

${ }^{10}$ Le don d'ovocyte entre mère et fille est plus rare puisqu'il faut que la mère soit suffisamment jeune. 
« Nous serions tentés de supposer que dans une société de droit maternel, comme celle des Trobriands, où tous les parents maternels sont considérés comme faisant partie du "même corps", tandis que le père est considéré comme un "étranger", on doit insister uniquement sur la ressemblance existant entre les enfants et leurs parents maternels. C'est cependant le contraire qui est vrai, et c'est le contraire qu'on fait ressortir avec une force et avec une insistance particulières. Ce n'est pas seulement un dogme domestique, pour ainsi dire, qu'un enfant ne ressemble jamais à sa mère, ni aux frères et sœurs ou tout autre parent de celle-ci, mais c'est faire preuve de mauvais goût et se rendre coupable d'une grave offense que de faire la moindre allusion à une ressemblance de ce genre. Mais il est naturel, juste et convenable qu'un homme ou une femme ressemble à son père ».

\section{0 Éloge du don « fraternel " ou « sororal» : et l'inceste psychique?}

Le don de gamètes entre parents est souvent présenté sur le mode idéalisé de la solidarité, comme si le fait qu'il ait lieu entre parents lui ôtait tout caractère sexuel. Or, il s'agit là d'un déni puisque les relations entre parents ne sont pas dénuées de sexualité quoique ceux-ci s'en défendent et que la règle de la prohibition de l'inceste l'interdise. Car, comme le souligne le psychanalyste Jean-Bertrand Pontalis : "L'inceste psychique ignore l'interdit »(2000 : 135). La sexualité n'est effectivement pas cantonnée au niveau physique de la rencontre des corps et de leurs substances, mais agit au niveau des mécanismes d'identification du psychisme ainsi que le montre l'analyse d'une pratique courante au CECOS qui a été instituée pour remédier au manque de dons.

Les couples ayant recours au don de sperme ou d'ovocyte sont invités à sensibiliser un homme ou une femme de leur entourage à leur projet contrarié d'enfant, afin qu'il ou elle se porte volontaire pour transmettre son sperme ou son ovocyte, don qui bénéficiera à d'autres couples que celui qui les a sollicités ; par cet acte qui a été fait à son intention mais qui ne lui reviendra pas directement, le couple demandeur verra son délai d'attente diminué et recevra à son tour les gamètes d'une personne qu'il ne connaît pas. Comme le remarque François Héritier (2009 : 194) dans un entretien avec Philippe Girard :

«La règle, c'est de donner à une femme un sperme qui n'est pas celui du donneur amené par le couple. Pourquoi? Sous les légitimations actuelles de la règle de l'anonymat, il me semble que se profile autre chose, qui relève du refus de l'inceste, de cette catégorie d'inceste particulier qui est la rencontre de deux consanguins dans une même femme».

Mais ce que ne souligne pas Françoise Héritier, c'est que cette mesure n'empêche pas l'inceste psychique dans la mesure où elle peut être génératrice de confusion pour le couple - et également pour celui ou celle qui donne - car, bien que la personne sollicitée ne soit pas à l'origine de leur enfant, puisque son sperme ou son ovocyte sera attribué à d'autres couples, le couple qui l'a sollicitée a néanmoins tendance à la 
considérer inconsciemment comme le géniteur ou la génitrice. Ainsi, parmi les cas rencontrés, une femme ayant demandé à son frère de venir donner son sperme au CECOS avait mal vécu sa grossesse qu'elle ressentait inconsciemment comme incestueuse, ne pouvant s'empêcher d'identifier le géniteur à son frère.

À ce sujet, on peut se demander si le don indirect de sperme ou d'ovocyte ne favorise pas la réalisation d'incestes fantasmatiques. En effet, la personne choisie par le couple est rarement un étranger du point de vue de la parenté puisqu'il s'agit souvent d'un parent proche, par exemple, dans le cas du don de sperme, un des frères de l'un des membres du couple, sinon un de leurs oncles. Il arrive, en outre, qu'au détour d'une conversation avec un couple ou un donneur, des situations incestueuses latentes émergent, par exemple lorsqu'une femme qui a sollicité un don de son frère souligne que son sperme a été reconnu comme « très bon » ou « très performant », et cela devant son mari, ou encore, lorsqu'un homme ayant donné son sperme à l'intention de sa sœur aînée, mentionne, lorsqu'il évoque son enfance, avoir toujours été amoureux de cette dernière.

Bien qu'aucune règle n'existe au CECOS sur la position des personnes apparentées pouvant être présentées comme donneurs indirects, lorsqu'un couple propose le père du côté de l'homme ou de la femme, le personnel médical refuse ce choix, plus ou moins conscient de son caractère incestueux. Or, reconnaître même implicitement la possibilité d'un tel inceste, c'est aussi admettre que ce dispositif apparemment non sexuel recouvre une certaine forme de sexualité.

La prétendue désexualisation du don d'ovocyte permettrait à une femme de recevoir l'ovocyte de la sœur de son mari; or, cette situation où la femme porte un enfant issu de la rencontre du sperme de son époux et de l'ovocyte de la sœur de celui-ci, participe de l'inceste sans pour autant être reconnu comme tel. En revanche, l'inceste serait plus immédiatement perçu s'il concernait un cas de figure symétrique à celui-ci du côté masculin, le don de sperme d'un homme à sa sœur, cas qui rappelle celui de Jeanine Salomone.

\section{L'inceste comme cumul d'identique : et l'inceste gémellaire?}

Françoise Héritier constate que l'inceste est toujours du côté du cumul de l'identique, entendu au sens substantiel, soit « la mise en rapport de deux identiques, de deux personnes ayant, même partiellement, une substance commune (Héritier 1994 : 12) que ce soit indirectement à travers un partenaire commun dans l'inceste du second type - comme deux sœurs, ou une fille et sa mère, ayant des relations sexuelles avec un même homme —, ou de manière directe dans l'inceste du premier type — entre un père et sa fille, une mère et son fils, ou un frère et sa sœur...

Françoise Héritier pense l'inceste de la même manière qu'elle pense la différence des sexes, soit à partir du corps féminin et non masculin. À la différence de Claude LéviStrauss (1967) pour lequel l'inceste fondateur est l'inceste père/fille, et à la différence de Sigmund Freud (1965, or. 1923; 1987, or. 1905 ; 1997, or. 1908) pour lequel l'inceste fils/mère est également important dans le complexe d'œdipe, cette femme anthropo- 
logue envisage l'inceste d'un point de vue féminin comme ancré dans le corps de la mère et de ses filles.

De plus, alors que l'inceste selon Claude Lévi-Strauss ou Sigmund Freud n'est envisagé que sur le mode hétérosexuel (père/fille, fils/mère, ou même frère/søur), Françoise Héritier met au jour l'inceste entre « deux sœurs et leur mère », inceste homosexuel par excellence. Même si Françoise Héritier n'emploie jamais cette expression dans son ouvrage Les deux sœurs et leur mère, lors d'un entretien donné avec le psychanalyste Jacques André (2001 : 224), elle reconnaît le caractère homosexuel de ce type d'inceste :

\begin{abstract}
«Vous signalez votre dette vis-à-vis de Reo Fortune, notamment d'une remarque de celui-ci dans un article de 1932 où il écrivait : "L'accent particulier qui a toujours été mis sur la relation qui existe entre la prohibition de l'inceste et la régulation du mariage a eu pour résultat qu'on a pratiquement toujours négligé de porter attention à l'inceste homosexuel" ».
\end{abstract}

Elle répond « [...] l'inceste homosexuel est pour moi un élément fondamental de cette combinatoire étonnante de l'inceste du deuxième type» (Héritier 2001 : 177).

L'inceste du « second type » entre deux sœurs et leur mère dérive de cet inceste premier que représente l'inceste mère-fille. Il est premier d'un point de vue psychique, ainsi que le montre la psychanalyse, mais également d'un point de vue physique, la fille et la mère partageant une «même forme ». Comme le déclare la romancière Nelly Arcan (2009 : 13) à propos de sa mère : «Elle et moi, on est dans le même bain, on vient du même moule ».

La notion de «forme » n'est pas étrangère à Françoise Héritier (2001 : 228) ainsi qu'en témoigne sa réponse au psychanalyste Jacques André quand il l'interroge sur l'inceste du second type :

« Si je considère que l'inceste du deuxième type des deux sœurs et leur mère est l'inceste fondamental, c'est parce qu'il y a la une triple conjonction de la "forme", de la nature (la consanguinité) et la chair. Cette conjonction existe aussi dans le rapport homme-fils. Pourtant j'ai placé en premier le rapport mère-fille parce qu'elles sont en plus issues l'une de l'autre, par l'accouchement. Dans le rapport père-fils, on peut bien dire aussi qu'ils sont issus l'un de l'autre, mais par une médiation qui est celle du corps de la mère; le fils est au premier chef l'enfant de cette mère-là. C'est pour cette raison que j'ai mis l'inceste mère-fille comme le modèle fondamental de l'inceste. Quant aux deux sœurs, elles sont issues du même corps de femme, dont elles ont la forme, la nature consanguine substantielle qu'elles touchent par l'intermédiaire d'un partenaire charnel commun. C'est un déplacement par rapport à la théorie de Lévi-Strauss où le couple paradigmatique sur le plan de la théorie de l'échange est celui du frère et de sœur ». 
On peut aussi retrouver cette idée d'identité de forme corporelle dans la notion de cumul de l'identique qui est au cœur de la théorie de l'inceste chez Françoise Héritier, même si elle l'emploie surtout dans le sens d'une conjonction de substance commune. Cette notion d'inceste me semble pertinente quand elle est étendue au-delà de l'identité de substance à la notion de forme corporelle, indépendamment même de la consanguinité, ce que semblait déjà relever de façon allusive Françoise Héritier (2001 : 220) dans cette affirmation sans pour autant la développer : « La place occupée par l'identité des formes corporelles, qui n'est pas l'identité consubstantielle de la consanguinité ». Cette idée de partage d'une même forme corporelle me semble essentielle pour définir l'inceste, aussi, je propose, pour ma part, le concept d' « inceste gémellaire », soit un type d'inceste qui peut s'appliquer indépendamment du sexe et de la parenté tant qu'il implique une relation réelle ou fantasmée de « gémellité » où la notion de ressemblance tient une place essentielle (Fortier 2019b).

\section{Une parenté de corps : et de ressemblances?}

De façon générale, il arrive que des personnes s'attachent à un enfant en raison du fait qu'il leur ressemble. C'est le cas par exemple d'une femme que j'ai rencontrée dans le cadre de mes recherches sur les ressemblances qui témoigne qu'elle a élevé les deux enfants de son frère jumeau comme les siens, notamment un des deux dont elle affirme « qu'il est sa copie conforme et qu'elle se reconnaît en lui ». Tout se passe comme si le fait de se voir dans le visage d'une autre personne à qui on ressemble créait un lien immédiat avec elle, une parenté.

Dans mes recherches sur les procréations médicalement assistées, la peur de se reconnaître dans les traits d'un enfant qui n'est pas le sien et par conséquent de ressentir des sentiments de type parental à son égard existe du côté des donneurs de gamètes. Rappelons qu'en France, le don est anonyme mais la plupart des donneurs que j'ai interrogés étaient inquiets à l'idée de croiser un enfant qui leur ressemblerait, sans doute du fait du sentiment d'inquiétante étrangeté (unheimlich) (Freud 1985, or. 1933) que cette rencontre produirait, mais surtout parce qu'ils seraient alors amenés à se demander si cet enfant n'était pas issu de leur don. Et nombreux sont ceux qui n'auraient jamais donné leur gamète si un tel don n'était pas anonyme, ne voulant pas avoir de relation avec l'enfant qui pourrait naître de ce don, affirmation qui cache la crainte de ressentir des sentiments de type parental à la vue de cet enfant, surtout s'il devait y avoir une ressemblance.

Cette idée apparaît aussi dans les témoignages de certaines donneuses d'ovocyte que j'ai rencontrées qui font le choix de donner anonymement en France plutôt que de façon directe dans d'autres pays par peur de s'attacher à l'enfant dans le cas où il leur ressemblerait. Ainsi, une femme dont j'ai recueilli le témoignage voulait aider son amie à avoir un enfant en lui donnant ses ovocytes de façon anonyme. Or, son amie souhaitait qu'elle fasse pour elle un don direct, ce qui est interdit en France, mais autorisé en Belgique. Cette femme fut alors confrontée à un dilemme, car si elle souhaitait aider son amie, elle ne voulait pas effectuer un don non anonyme de crainte que la ressemblance éventuelle entre cet enfant et son propre enfant éveille chez elle le sentiment d'en être la mère : « J'ai pensé, si elle a une fille qui ressemble à la mienne, 
est ce que je ne vais pas me sentir un peu comme sa maman? Finalement, comme mon amie refusait le don anonyme, j'ai donné toute seule au CECOS à la liste de ceux qui n'ont pas eu la chance de trouver une donneuse ».

Il est tout à fait significatif de constater que ce n'est pas le lien biologique proprement dit qui est ici considéré comme à l'origine du sentiment d'être apparenté à l'enfant mais plutôt le lien de ressemblance. Dans ce cas, la femme appréhende la ressemblance entre l'enfant né de son don et son propre enfant plutôt que la ressemblance avec ellemême, parce que, davantage observable, elle l'amène à s'interroger sur l'importance de la ressemblance dans la naissance du sentiment parental.

Cette inquiétude exprimée ici par une donneuse est aussi ressentie par des femmes qui ont recours au don d'ovocyte. Si le don venait d'une proche, sœur, belle-sœur ou amie, comme cela est autorisé dans certains pays et l'était encore il y a quelque temps en France, les mères craignent que leur enfant ne ressemble à cette personne et que celle-ci s'y attache au point de se comporter avec lui comme sa mère. Cet argument les amène à choisir le don anonyme en France plutôt que le don direct envisageable dans d'autres pays d'Europe, et ce malgré la plus grande lenteur de la procédure et donc le plus faible taux de succès au final.

\section{Conclusion}

Si l'enfantement est valorisé dans la plupart des sociétés comme un pouvoir spécifiquement féminin ainsi que l'a bien mis en évidence Françoise Héritier (1996), mes recherches montrent qu'un pouvoir masculin l'est plus encore en amont, celui de l'engendrement, celui-ci précédant et déterminant l'enfantement dans la réalité comme dans la pensée compte tenu du rôle fécondant du sperme, substance visible à la différence de l'ovocyte. Aussi, il me semble que psychanalyse et anthropologie convergent relativement au constat que le primat du masculin au niveau psychique et social s'enracine dans la différence sexuée, qu'elle soit lue dans une différence d'organes, avoir ou non un pénis, ou une différence de substances, avoir ou non du sperme, signes quasi équivalents de la puissance sexuelle masculine.

Mes propres travaux, à la différence de ceux de Françoise Héritier, tendent à prendre en considération non seulement le corps féminin, mais masculin ${ }^{11}$, non seulement l'enfantement mais l'engendrement, non seulement l'utérus - ou le ventre pour reprendre le terme de Marcela Iacub (2004) —, mais le sperme, et non seulement la procréation mais l'érotisme, érotisme qui, selon mes recherches, caractérise tout autant la femme que sa capacité procréative.

Et si le corps est important dans la détermination du genre (Fortier 2021f), il l'est aussi dans la représentation que l'on se fait de la parenté. À la constatation de ressemblances est rapportée spontanément non seulement une parenté mais aussi un « sentiment affectif » censé lui être coextensif. On souligne peu l'importance de cette dimension dans l'émergence supposée du sentiment de parenté mais, pour le sens commun, c'est bien la ressemblance qui fait la parenté. La ressemblance est à la fois

\footnotetext{
${ }^{11}$ C'est le cas également de l'anthropologue américaine Marcia Inhorn (2009) relativement aux procréations médicalement assistées dans le monde arabe.
} 
au cœur de la problématique de l'interdit de l'inceste en tant qu'il lui est associé un sentiment de parenté, qu'au fondement de l'inclination incestueuse dans la mesure où celui qui me ressemble peut apparaître comme «un autre soi-même» désirable, inceste que je conceptualise sous le terme «d'inceste gémellaire». Le corps demeure donc éminemment important pour penser le genre et la parenté, même si le genre n'est pas le sexe, tout comme la parenté n'est pas la ressemblance.

\section{Bibliographie}

Altorki, Soraya (1980) «Milk-Kinship in Arab Society : An Unexplored Problem in the Ethnography of Marriage », Ethnology, vol. 19, University of Pittsburg, États-Unis, p. 233-244.

Arcan, Nelly, Paradis, clef en main, Coups de tête, Montréal, 2009.

Bettelheim, Bruno, Les Blessures symboliques. Essai d'interprétation des rites d'initiation, Gallimard, Paris, 1971.

Butler, Judith, Trouble dans le genre. Pour un féminisme de la subversion, préface d'Éric Fassin, traduction de Cynthia Kraus, La Découverte, Paris, 2005, $1^{\text {re }}$ édition 1990.

De Rougemont, Denis, L'amour et l'Occident, Plon, Coll. «10/18», Paris, 1972.

Devereux, Georges, Baubo, la vulve mythique, Payot, Coll. «Petite Bibliothèque », Paris, 2011, $1^{\text {re }}$ édition 1983.

Dumont, Louis (1966) «Vers une théorie de la hiérarchie», postface, in Dumont, Louis, Homo hierarchicus, Le système des castes et ses implications, Gallimard, Coll. « Tel », Paris, p. 396-403.

Fortier, Corinne (1998) «Rapports sociaux de sexe et représentation de la genèse physiologique des enfants dans le Touat-Gourara (Sahara algérien) », in Lacoste-Dujardin, Camille; Virolle, Marie (dir.), Femmes et hommes au Maghreb et en immigration, la frontière des genres en question, Publisud, France, p. 47-69.

Fortier, Corinne (2001) " "Le lait, le sperme, le dos. Et le sang?". Représentations physiologiques de la filiation et de la parenté de lait en islam malékite et dans la société maure de Mauritanie ", Les Cahiers d'Études Africaines, vol. XL(1), No 161, éditions de l'EHESS, France, p. 97-138.

Fortier, Corinne (2004a) «Séduction, jalousie et défi entre hommes. Chorégraphie des affects et des corps dans la société maure », in Héritier, Françoise ; Xanthakou, Margarita (dir.), Corps et affects, Odile Jacob, France, p. 237-254.

Fortier, Corinne (2004b) «"Ô langoureuses douleurs de l'amour". Poétique du désir en Mauritanie», in Demeuldre, Michel (dir.), Sentiments doux-amers dans les musiques du monde, L'Harmattan, Coll. " Logiques sociales, musique et champ social», France, p. 15-25.

Fortier, Corinne (2005) «Le don de sperme et le don d'ovocyte ou "trois font un". Sexualité, inceste et procréation », in Bidou, Patrice; Galinier, Jacques; Juillerat, Bernard (dir.), Anthropologie et psychanalyse : regards croisés, éditions de l'EHESS, France, p. 59-80.

Fortier, Corinne (2007) «Blood, Sperm and the Embryo in Sunni Islam and in Mauritania : Milk Kinship, Descent and Medically Assisted Procreation », Body and Society, vol. 13, No 3, Islam, Health and the Body, Tober, Diane; Budiani, Debra (dir.), Sahe Journals, États-Unis, p. 15-36.

Fortier, Corinne (2009) «Quand la ressemblance fait la parenté », in Porqueres i Gené, Enric (dir.), Défis contemporains de la parenté, éditions de l'EHESS, Coll. « Cas de figure», France, p. 251-276.

Fortier, Corinne (2010) «La barbe et la tresse. Marqueurs de la différence sexuée (société maure de Mauritanie)", Les Cahiers du Laboratoire d'Anthropologie Sociale, vol. 6, Poils et sang, Karadimas, Dimitri (dir.), L'Herne, France, p. 94-104.

Fortier, Corinne (2011a) «Women and Men put Islamic Law to their Own Use : Monogamy versus Secret Marriage in Mauritania », in Badran, Margot (dir.), Gender and Islam in Africa. Rights, Sexuality and Law, Woodrow Wilson Press, États-Unis, p. 213-232.

Fortier, Corinne (2011b) «Filiation versus inceste en islam : parenté de lait, adoption, PMA, reconnaissance de paternité De la nécessaire conjonction du social et du biologique», in Bonte, Pierre; Porqueres, Enric; Wilgaux, Jérôme (dir.), L'argument de la filiation aux fondements des sociétés méditerranéennes et européennes, éditions de la MSH, France, p. 225-248. 
Fortier, Corinne (2011c) «"Des gamètes de couleur" : phéno-type, race ou ethnie?» L'Autre. Cliniques, cultures et sociétés, Revue transculturelle. Parentalités, vol. 12, No 3, éditions La pensée sauvage, France, p. 289-303.

Fortier, Corinne (2011d) «Tentation eugénique et ethnicisation biologique de la différence physique : le cas du don de gamètes », in Rude-Antoine, Edwige; Pievic, Marc (dir.), Éthique et famille, t. 2, L'Harmattan, Coll. «Éthique en contextes », France, p. 207-221.

Fortier, Corinne (2012a) «Sculpter la différence des sexes. Excision, circoncision et angoisse de castration (Mauritanie) », in Lachheb, Monia (dir.), Penser le corps au Maghreb, Karthala/IRMC, Coll. "Hommes et Sociétés », France, p. 35-66.

Fortier, Corinne (2012b) «De troublantes ressemblances : un sentiment de parenté », Corps, No 10, Corps des affects, corps en migrations, Diasio, Nicoletta; Vinel, Virginie (dir.), éditions du CNRS, France, p. $105-113$.

Fortier, Corinne (2014a) «La question du "transsexualisme" en France. Le corps sexué comme patrimoine», Les Cahiers de droit de la santé, No 18, Corps et Patrimoine, Nicolas, Guylène (dir.), éditions LEH, France, p. 269-282.

Fortier, Corinne (2014b) «Inscribing Trans and Intersex People in the Dominant Binary Categories of Gender », Etropic, vol. 13, No 2, Value, Transvaluation and Globalization, Dalsgaard, Steffen; Otto, Ton (dir.), James Cook University, Australie, p. 1-13. https://journals.jcu.edu.au/etropic/issue/view/158

Fortier, Corinne (2017a) «Intersexués, le troisième genre en question en France et au-delà », Socio. La nouvelle revue des sciences sociales, No 9, Combien de sexes?, Coburn, Elaine (dir.), éditions de la Fondation Maison des Sciences de l'Homme, France, p. 91-106. https://journals.openedition.org/socio/3036

Fortier, Corinne (2017b) «L'insémination avec donneur. Corps féminin, virilité procréative et fantasme d'adultère», Spirale, No 84, L'Assistance Médicale à la Procréation : de l'extraordinaire aux risques du banal, Canneaux, Marion; Missonier, Sylvain (dir.), éditions Érès, France, p. 49-54.

Fortier, Corinne (2018a) «The expences of Love : Seduction, Poetry and Jealousy in Mauritania», in Fortier, Corinne; Kreil, Aymon; Maffi, Irene (dir.), Reinventing Love? Gender, Intimacy and Romance in the Arab World, Peter Lang, Coll. «Middle East, Social and Cultural Studies - Études culturelles et sociales sur le Moyen-Orient », Suisse, p. 47-69.

Fortier, Corinne (2018b) «Milk, Breast», in Callan, Hilary (dir.), The International Encyclopedia of Anthropology, John Wiley, Angleterre. doi : 10.1002/9781118924396.wbiea1433

Fortier, Corinne (2018c) «The Gendered Gift of Gamets : Sexuality, Incest and Procreation », in Flood, Roisin Ryan; Gunnarsson Payne, Jenny (dir.), Transnationalising Reproduction : Third Party Conception in a Globalized World, Routledge, États-Unis, p. 48-58.

Fortier, Corinne (2018d) Le danger y a toujours! Thomas, pêcheur de Penmar'ch, film documentaire de 14 mns. https://www.canal-u.tv/video/ehess/le_danger_y_a_toujours_thomas_pecheur_de_penmar_ch. 48009

Fortier, Corinne (2018e) Ben oui c'est encore les femmes! Danièle, femme de marin, film documentaire de 14 mns. https://www.canal-u.tv/video/ehess/ben_oui_c_est_encore_les_femmes.47467

Fortier, Corinne (2019a) «Germaine Tillion, une femme ethnologue engagée», in Yacine, Tassadit (dir.), Germaine Tillion, une ethnologue engagée, Non Lieu, France, p. 171-188.

Fortier, Corinne (2019b) «Inceste gémellaire, deuil et mélancolie créatrice. De la transidentité à l'œuvre de Pierre Molinier et d'Annie Ernaux », L'Autre, vol. 20, No 1, Françoise Héritier : leçons apprises, Mestre, Claire; Moro, Marie-Rose (dir.), éditions La pensée sauvage, France, p. 51-61.

Fortier, Corinne (2019c) «Sexualities : Transexualities : Middle East, North Africa, West Africa», Joseph, Suad (dir.), Encyclopedia of Women and Islamic Cultures (EWIC), Supplement 20, Brill, Pays-Bas. http://dx.doi.org/10.1163/1872-5309_ewic_COM_002185

Fortier, Corinne (2019d) «Procréations médicalement assistées, sexualité et religions. Circulation sexuelle des gamètes en islam et dans les autres monothéismes », Ateliers d'anthropologie, No 46, Corps perdu, corps retrouvé : cohésion, partitions, variétés, Jamard, Jean-Luc ; Lacaze, Gaelle; Xanthakou, Margarita (dir.), éditions du Laboratoire d'ethnologie et de sociologie comparative, France. http://journals.openedition.org/ ateliers/11063

Fortier, Corinne (2020a) «Reconstruction clitoridienne, excision et circoncision. Variations autour d'un sexe féminin phallique», Droits et Cultures, No 79, Réparer les corps et les sexes, vol. 1, Excision, circoncision, et reconstruction clitoridienne, Fortier, Corinne (dir.), éditions L'Harmattan, France p. 2976. https://journals.openedition.org/droitcultures/5977 
Fortier, Corinne (2020b) «Religious and non-religious issues of medically assisted reproduction in France. Sexuality, Incest and Descent », Reproductive BioMedicine and Society (RBMS), No 144, Mathieu, Séverine; Rapp, Rayna (dir.), Elsevier, États-Unis. https://www.rbmsociety.com/article/S2405-6618(20)30026$5 /$ fulltext

Fortier, Corinne (2021a) «Passion Love, Masculine Rivalry and Arabic Poetry in Mauritania », in Mayer, Claude-Helene; Vanderheiden, Elisabeth (dir.), International Handbook of Love. Transcultural and transdisciplinary perspectives, chapter 41, Springer International, États-Unis, p. 769-788.

Fortier, Corinne (2021b) «Le désir poétisé ou le goût des Fleurs du mal (poésie maure / poésie arabe antéislamique). Poetised Desire or the Taste of Flowers of evil in Moorish and Arabic Pre-Islamic Poetry », Anthropology of the Middle East, vol. 16, No 2, winter, Poetised Love : Affects, Gender and Society. L'amour poétisé : affects, genre et sociétés, Fortier, Corinne (dir.), Berghahn Journals, États-Unis, p. 3356. doi : 110.3167/ame.2021.160202

Fortier, Corinne (2021c) «Introduction. L'amour poétisé : genre, plaisir et nostalgie dans la poésie arabe et persane masculine, féminine et homoérotique. Gender, Pleasure and Nostalgia in Masculine, Feminine and Homoerotic Arabic and Persian Poetry ", Anthropology of the Middle East, vol. 16, No 2, winter, Poetised Love: Affects, Gender and Society. L'amour poétisé : affects, genre et sociétés, Fortier, Corinne (dir.), Berghahn Journals, États-Unis, p. 1-32. doi : 10.3167/ame.2021.160201

Fortier, Corinne (2021d) «Des "certificats de virginité" aux hyménoplasties en France», Médecine/Sciences, vol. 37, No 4, Inserm, France, p. 392-395. https://www.medecinesciences.org/fr/articles/ medsci/abs/2021/04/msc200336/msc200336.html

Fortier, Corinne (2021e) «Chirurgies sexuelles. Du corps transformé à l'identité retrouvée », in Fortier, Corinne (dir.), Le corps de l'identité. Transformations corporelles, genre, et chirurgies sexuelles, Karthala, France, à paraître.

Fortier, Corinne (2021f) «Virginité féminine/défloration masculine. "Certificats de virginité"/chirurgies de revirginisation, in Fortier, Corinne (dir.), Le corps de l'identité. Transformations corporelles, genre et chirurgies sexuelles, Karthala, France, à paraître.

Fortier, Corinne (2022a) «Gavage, genre et sexualité dans la société maure de Mauritanie », in Tinat, Karine (dir.), Alimentación y sexualidad, à paraître.

Fortier, Corinne (2022b) «Absence des hommes et empowerment des femmes de marins (Finistère, Vendée) », in Charpentier, Emmanuelle; Grenier, Benoît (dir.), Le temps suspendu. Une histoire des femmes mariées par-delà le silence et l'absence, Presses Universitaires du Midi, France, à paraître.

Fortier, Corinne; Kreil, Aymon; Maffi, Irene (2016) «The Trouble of Love in the Arab World : Romance, Marriage, and the Shapping of Intimate Lives", The Arab Studies Journal, Special Section : Love in the Arab World, vol. 24, No 2, Georgetown University, États-Unis, p. 96-101.

Fortier, Corinne; Kreil, Aymon; Maffi, Irene (2018) «Introduction. Reinventing Love? Gender, Intimacy and Romance in the Arab World », in Fortier, Corinne; Kreil, Aymon; Maffi, Irene (dir.), Reinventing Love? Gender, Intimacy and Romance in the Arab World, Peter Lang, Coll. "Middle East, Social and Cultural Studies - Études culturelles et sociales sur le Moyen-Orient », Suisse, p. 9-32.

Freud, Sigmund, Totem et tabou, Payot, Coll. «Petite bibliothèque », France, 1965, $1^{\text {re }}$ édition 1923.

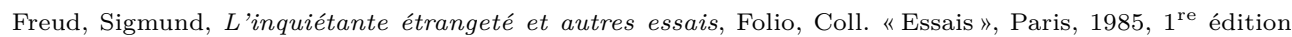
1933.

Freud, Sigmund, Trois Essais sur la théorie sexuelle, Gallimard, Coll. «Folio Essais », Paris, 1987, $1^{\text {re }}$ édition 1905.

Freud, Sigmund, La vie sexuelle, PUF, Paris, 1997, $1^{\text {re }}$ édition 1908.

Godelier, Maurice, La production des grands hommes : pouvoir et domination masculine chez les Baruya de Nouvelle Guinée, Fayard, Paris, 1982.

Green, André (1971) «De la bisexualité au gynocentrisme», préface, in Bettelheim, Bruno, Les Blessures symboliques. Essai d'interprétation des rites d'initiation, Gallimard, Paris, p. 213-234.

Héritier, Françoise (1985) «Le Sang du guerrier et le sang des femmes : notes anthropologiques sur le rapport des sexes ", Les Cahiers du Grif, No 29, L'Africaine : sexe et signe, GRIF, Belgique, p. 7-21.

Héritier, Françoise, Les deux sœurs et leur mère. Anthropologie de l'inceste, Odile Jacob, France, 1994.

Héritier, Françoise, Masculin/Féminin. La pensée de la différence, Odile Jacob, France, 1996. 
Héritier, Françoise (1997) «Quelques propos tenus lors de l'assemblée générale de l'AFA 22 mai 1996 », Journal des anthropologues, Anthropologie et psychanalyse, vol. 71, No 4, Association Française des Anthropologues, France, p. 9-16.

Héritier, Françoise (2001) «Inceste et substance. Edipe, Allen, les autres et nous. Entretien avec Jacques André », in André, Jacques (dir.), Incestes, PUF, Coll. "Petite bibliothèque de psychanalyse », France, p. 91-133.

Héritier, Françoise, Maculin/féminin II. Dissoudre la hiérarchie, Odile Jacob, Paris, 2002.

Héritier, Françoise, Une pensée en mouvement, Odile Jacob, Paris, 2009.

Iacub, Marcela, L'empire du ventre, Fayard, Coll. « Histoire de la pensée», Paris, 2004.

Inhorn, Marcia, Reconceiving the Second Sex : Men, Masculinity, and Reproduction, Berghahn Books, Oxford/New York, 2009.

Lacan, Georges, Le Séminaire. Livre XI, Les quatre concepts fondamentaux de la psychanalyse, Seuil, Paris, 1973, $1^{\text {re }}$ édition 1964.

Lévi Strauss, Claude, Les Structures élémentaires de la parenté, Paris, Mouton, 1967.

Malinowski, Bronislaw, La vie sexuelle des sauvages, Payot, Paris, 1930.

Mulvey, Laura (1975) «Visual Pleasure and Narrative Cinema», Screen, vol. 16, No 3, Oxford University Press, Angleterre, p. 6-18.

Pontalis, Jean-Bertrand, Fenêtres, Gallimard, Paris, 2000.

Rivière, Joan (1994) «La féminité en tant que mascarade», in Hamon, Marie-Christine (dir.), Féminité Mascarade. Études psychanalytiques, Le Seuil, Coll. «Champ freudien», France, p. 197-213, $1^{\text {re }}$ édition 1929.

Salomone, Jeanine, Je l'ai tant voulu. Maman à 62 ans, J.-C. Lattès, Paris, 2002.

Tillion, Germaine, La traversée du Mal. Entretien avec Jean Lacouture, Arléa, Paris, 1997. 